place. A negative environmental trend would imply some deterioration of standardized environment within station...

Although not all possible biases were removed, the results do seem to indicate that the desired genetic progress is being made in Swedish pig breeding.

\author{
OBSERVED RESPONSE TO INDIVIDUAI, SELECTION \\ OF BOARS OVER A PERIOD OF ELEVEN YEARS

\section{OLLIVIER} \\ Département de Génétique animale, \\ Centre national de Recherches agronomiques, I.N.R.A., \\ 78350 Jouy-en-Josas, France
}

Starting from ten Large White "founder" boars, put into service in november $\mathbf{x} 965$, ten successive yearly boar generations have been selected on a performance-test index, equal to $0.01 \mathrm{ADG}-0.5 \mathrm{BF}, \mathrm{ADG}$ being average daily gain (g) from 30 to $80 \mathrm{~kg}$ liveweight and $\mathrm{BF}$ being the average of six backfat measurements at $80 \mathrm{~kg}$ liveweight. Selection responses were estimated for growth rate, feed efficiency, carcass and meat quality traits and nasal turbinates development. The data analyzed, which include 1604 female and 1284 castrated male progeny from ror boars, show linear genetic trends which are, as a rule, relatively larger over sire generations than over dam cohorts. The antiual sire genetic trend in lean tissue growth rate is $6 \mathrm{~g}$ per day (2.6 p. Ioo), which is twice the dam trend. In lean tissue feed conversion, the annual genetic gain, which an only be estimated for sires, is $0.2 \mathrm{~kg}$ feed per $\mathrm{kg}$ lean tissue (I.9 p. IOO). Correlated responses in meat quality traits indicate a tendency towards a paler meat colour, but conflicting sire and dam trends are observed for $\mathrm{pH} 2.4$ and water-holding capacity. Unfavourable sire and dam trends are observed for nasal turbinates development, which indicate a greater susceptibility to atrophic rhinitis as a consequence of the selection practised in this experiment.

\title{
ERFAHRUNGEN MIT UND ERGEBNISSE DER INDEXSELEKTION BEIM SCHWEIN UNTER FELDBEDINGUNGEN
}

\author{
W. PESCHKE UND G. AVERDUNK \\ Bayerische Landesanstalt für Tierzucht, D-8oII Grub (B.D.R.)
}

Aug Grund der Nachkommenleistung von I637 Ebern wurde der vorgeschätzte Zuchtwert mit dem der Nachkommen verglichen. Die Selektionsintensität der ausgewählten Eber betrug I.2IS bzw. I.59s für den vorgeschätzten Zuchtwert. Die Korrelation zwischen den Zuchtwerten betrug. тo -... I2. Für die Merkmale des Schlachtkörpers sind die Korrelationen höher als für die Mastleistung.

Für den Index ergibt sich zwischen Eigen- und Nachkommen- leistung eine Korrelation von .55. Die Rangierung der Eber nach verschiedenen Auswahlverfahren zeight, dass bei der Selektion nach Index auch die Nachkommen den höchsten Index erbrachten.

\section{ZUCHTWERTSCHÄTZUNG VON JUNGSAUEN UND SAUEN UNTER PRAKTISCHEN BEDINGUNGEN}

\author{
I. GAJIK und L. I.AZAREVIC \\ Landrwtchaftliches Fakultät, \\ Zemun, Jugoslawien
}

Die Zuchtwertschätzung von Jungsauen kann von zwei Gesichtspunkten betrachtet werden. Diese sind zum einen die Genauigkeit und zum anderen die Frage, in welchem Alter die Zuchtwertschätzung vorgenommen werden sollte. Werden beide Ziele erreicht, z.B. ausreichende Genauigkeit bei niedrigem Alter, so resultiert hieraus ein grösserer genetischer Fortschritt der einzelnen Populationen. Es ist bekannt, dass dies kein Problem darstellt und leicht zu lösen ist. Es werden einige Methoden der Schätzung des Zuchtwertes von Jungsauen und Sauen auf 
grossen Schweinezuchtbetrieben mit hoher Merzquote diskutiert. Die Daten, auf denen die Zuchtwertschätzung basiert, wurden auf den Betrieben gesammelt. Bei einigen Betrieben werden die Daten nicht nur für die Selektion verwendet.

Der Zuchtwert von Jungsauen wird mit Hilfe eines Selektions-index geschätzt, der die Merkmale Rückenspeckdicke und Lebendgewicht beinhaltet. Die Rückenspeckdicke ist auf das Gewicht von Ioo kg sowie auf ein Alter von 200 Tagen korriegiert. Es wurden Heritabilitätsschätzungen auf zwei Betrieben durchgeführt. Die Werte betrugen für Betrieb A o, I9 und o, 25 sowiefür Betrieb $B$ o,44 und o,55. Die Korrelationen zwischen den Selektionsindizes und dem Gesamtgenotyp betrugen $r_{\text {IH }}=0,33$ und 0,29 .

Der Zuchtwert der Sauen wird anhand eines Selektıonsindex geschätzt, der die Zahl der Ferkel bei Geburt sowie im Alter von 28 Tagen sowie das Wurfgewicht bei Geburt und im Alter von 28 Tagen einschliesst. Der Selektionsindex wurde für die ersten und folgenden Würfe aufgestellt. Die Heritabilitäts-schätzungen für die in den Indizes eingeschlossenen Eigenschaften schwanken von 0,03 bis $0,3^{8}$. Die Selektionsindizes werden auf der Grundlage von Differenzen der gleitenden Durchschnitte gebildet. Die Korrelation zwischen deni Selektionsindex und dem Gesamtgenotyp für Erstlingswürfe betrug $r_{I H}=0,47$ und für nachfolgende Würfe $0,6 \mathrm{I}$.

\section{FRESE, E. BRUNS und P. GLODEK}

Institut $f$. Tierzücht und Haustiergenetik, Göttingen, B.R.D.

Für die Zuchtwertschätzung von Jungebern wird neben der Eigenleistung auch die Völlund Halbgeschwisterleistung als Informationsquelle verwendet. Die Leistungen der Halbgeschwister können jedoch nur dann herangezogen werden wenn diese aus Paarungen des Vaters an eine Zufallsstichprobe von Sauen erstellt werden.

Der mittlere Zuchtwert der ar einen E,ber angepaarten Sauen liegt in zwei Zuchtverbänden bei xo5 bzw. I Io Indexpunkten und hat eine Standard-abweichung von 9.9 bzw. 9.6 Indexpunkten.

Die Nachkommenleistung muss daher für den Einfluss des Zuchtwertes der witter korrigiert werden. Für diese Korrektur werden Regressions-koeffizienten geschätzt.

Die Korrektur kann anhand von 2 Methoden durchgeführt werden:

I. Lineare Korrektur der Halbgeschwisterinformationen mit den geschätzten Regressions koeffizienten.

2. Eiribeziehung der Leistung der Mutter in den Index des Ebers.

CORRELATION BETWEEN PERFORMANCE TESTS OF BOARS ON AUCTION SAIES AND STATION TEST OI SIBS

\section{H. SCHULTE-COERNE and D. L. SIMON}

Institute of Animal breeding, University of Bonn, Laboratorr of Genetics D.53oo Bonn, Western Germany

Product moment correlations have been computed between gain per day of age and ultrasonic backfat thickness of boars on auction sales and the respective mearis of sibs tested for growth and carcass traits on station.

Correlations are compared to expected correlations estimated from genetic parameters reported in literature.

Though generally low, observed and expected correlations irvolving gain per day of age are in good agreement.

Observed correlations involving ultrasonic backfat thickness however do not fit the relatively favourable expected correlations. This may be due to effects of selection or low standard of ultrasonic measurement. 